\title{
Barrier Options and a Reflection Principle of the Fractional Brownian Motion
}

\author{
Ciprian Necula \\ DOFIN \\ Academy of Economic Studies \\ Bucharest, Romania \\ Email: ciprian.necula@fin.ase.ro \\ cipnec@yahoo.com
}

First draft: September 26, 2003

\begin{abstract}
$\underline{\text { Abstract }}$
The purpose of this paper is to obtain the price of the barrier options in a fractional Brownian motion environment in the special case of zero interest rate. As a consequence we derive a reflection principle for the fractional Brownian motion.
\end{abstract}




\section{Introduction}

If $0<H<1$ the fractional Brownian motion ( $\mathrm{fBm}$ ) with Hurst parameter $H$ is the continuous Gaussian process $\left\{B_{H}(t), t \in \mathbb{R}\right\}, B_{H}(t)=0$ with mean $E\left[B_{H}(t)\right]=0$ and whose covariance is given by:

$$
C_{H}(t, s)=E\left[B_{H}(t) B_{H}(t)\right]=\frac{1}{2}\left\{|t|^{2 H}+|s|^{2 H}-|t-s|^{2 H}\right\}
$$

If $H=\frac{1}{2}$ then $B_{H}(t)$ coincides with the standard Brownian motion $B(t)$.

The fractional Brownian motion is a self-similar process meaning that for any $\alpha>0 \quad B_{H}(\alpha t)$ has the same law as $\alpha^{H} B_{H}(t)$.

The constant $\mathrm{H}$ determines the sign of the covariance of the future and past increments. This covariance is positive when $H>\frac{1}{2}$, zero when $H=\frac{1}{2}$ and negative when $H<\frac{1}{2}$. Another property of the fractional Brownian motion is that for $H>\frac{1}{2}$ it has long-range dependence.

The self-similarity and long-range dependence properties make the fractional Brownian motion a suitable tool in different applications like mathematical finance.

Since for $H \neq \frac{1}{2}$ the fractional Brownian motion is neither a Markov process, nor a semimartingale, we cannot use the usual stochastic calculus to analyze it. Worse still after a pathwise integration theory for fractional Brownian motion was developed (Lin (1995), Decreusefond and Ustunel (1999)) it was proven that the market mathematical models driven by $B_{H}(t)$ could have arbitrage (Rogers (1997)). The fractional Brownian motion was no longer considered fit for mathematical modeling in finance. However after the development of a new kind of integral based on the Wick product (Duncan, Hu and Pasik-Duncan (2000), Hu and Oksendal (2000)) called fractional Ito integral, it was proved (Hu and Oksendal (2000)) that the corresponding Ito type fractional Black-Schools market has no arbitrage. There are some other ways of defining the fractional Ito integral. See for example Alos, Mazet and Nualart (2001), Alos and Nualart (2002), Perez-Abreau and Tudor (2002) or Bender (2002). A review of the results concerning the fractional Ito integral can be found in Necula (2002) or Necula (2003).

In the paper of $\mathrm{Hu}$ and Oksendal (2000) a formula for the price of a European option at $t=0$ is derived and the formula is extended for every $t \in[0, T]$ in the paper of Necula (2002). Bender (2003) generalized it for the case of a non constant but deterministic volatility, using not the quasi-conditional expectation but linear fractional BSDE.

The purpose of this paper was to derive the price of barrier options in a fractional Brownian motion environment. But so far we only managed to obtain the price of barrier option for the case of zero interest rate. These results are not very appealing for the finance, but they can be used to obtain for the fractional Brownian motion something that can be called the reflection principle. 


\section{A Reflection Principle for the Fractional Brownian Motion} a stock.

Consider a fractional Black-Scholes market with a money market account and

Under the risk-neutral measure $(P)$ we have that the stock price satisfies the equation:

$$
d S(t)=r S(t) d t+\sigma S(t) d B_{H}(t), \quad S(0)=S>0,0 \leq t \leq T
$$

where $r$ represent the constant riskless interest rate.

We will denote by $\widetilde{E}_{t}[$.$] the quasi-conditional expectation (Hu and Oksendal$ (2000)) with respect to the risk-neutral measure.

We know that the price of a $\mathscr{F}_{T}{ }^{H}$ - measurable contingent claim $F$ is given by (Necula (2002)):

$$
F(t)=e^{-r(T-t)} \widetilde{E}_{t}[F]
$$

We consider the contingent claims:

- Binary call and put with strike $K: B C=1_{\{S(T)>K\}}, \quad B P=\mathbb{1}_{\{S(T)<K\}}$

- Gap call and put with strike $K: G C=S(T) 1_{\{S(T)>K\}}, G P=S(T) \mathbb{1}_{\{S(T)<K\}}$

\section{Lemma 2.1}

We have that:

$$
\begin{gathered}
B C(t, S(t), K)=e^{-r(T-t)} N\left(d_{2}\right), B P(t, S(t), K)=e^{-r(T-t)} N\left(-d_{2}\right) \\
G C(t, S(t), K)=S(t) N\left(d_{1}\right), G P(t, S(t), K)=S(t) N\left(-d_{1}\right)
\end{gathered}
$$

where

$$
\begin{aligned}
& d_{1}=\frac{\ln \left(\frac{S(t)}{K}\right)+r(T-t)+\frac{\sigma^{2}}{2}\left(T^{2 H}-t^{2 H}\right)}{\sigma \sqrt{T^{2 H}-t^{2 H}}} \text { and } \\
& d_{2}=\frac{\ln \left(\frac{S(t)}{K}\right)+r(T-t)-\frac{\sigma^{2}}{2}\left(T^{2 H}-t^{2 H}\right)}{\sigma \sqrt{T^{2 H}-t^{2 H}}}
\end{aligned}
$$

and $N(\cdot)$ is the cumulative probability of the standard normal distribution. 
Proof:

See the proof of Theorem 4.2 in Necula (2002).

We will make the following notations:

$$
\begin{aligned}
& \tau_{L}:=\inf \{t \mid S(t)=L\} \\
& m^{S}(T):=\inf _{0<t<T} S(t) \\
& M^{S}(T):=\sup _{0<t<T} S(t)
\end{aligned}
$$

First we will analyze the case of no drift in 2.1 (i.e $r=0$ )

We know that $S(t)=S \exp \left\{-\frac{\sigma^{2}}{2} t^{2 H}+\sigma B_{H}(t)\right\}$

\section{Theorem 2.1}

Consider that $r=0$.

1. If $K>L, S>L$ and $t<\tau_{L}$ then

$$
\widetilde{E}_{t}\left[1_{\left\{S(T)>K, m^{S}(T)>L\right\}}\right]=N\left(a_{1}\right)-\frac{S(t)}{L} N\left(a_{2}\right)
$$

where $a_{1}=\frac{\ln \left(\frac{S(t)}{K}\right)-\frac{\sigma^{2}}{2}\left(T^{2 H}-t^{2 H}\right)}{\sigma \sqrt{T^{2 H}-t^{2 H}}}$ and $a_{2}=\frac{\ln \left(\frac{H^{2}}{L S(t)}\right)-\frac{\sigma^{2}}{2}\left(T^{2 H}-t^{2 H}\right)}{\sigma \sqrt{T^{2 H}-t^{2 H}}}$

2. If $S>L$ and $t<\tau_{L}$ then

$$
\widetilde{E}_{t}\left[\mathbb{1}_{\left\{\tau_{L}>T\right\}}\right]=\widetilde{E}_{t}\left[\mathbb{1}_{\left\{m^{S}(T)>L\right\}}\right]=N\left(b_{1}\right)-\frac{S(t)}{L} N\left(b_{2}\right)
$$

where $b_{1}=\frac{\ln \left(\frac{S(t)}{L}\right)-\frac{\sigma^{2}}{2}\left(T^{2 H}-t^{2 H}\right)}{\sigma \sqrt{T^{2 H}-t^{2 H}}}$ and $b_{2}=\frac{\ln \left(\frac{L}{S(t)}\right)-\frac{\sigma^{2}}{2}\left(T^{2 H}-t^{2 H}\right)}{\sigma \sqrt{T^{2 H}-t^{2 H}}}$

3. If $K<L, S<L$ and $t<\tau_{L}$ then

$$
\widetilde{E}_{t}\left[{ }_{\left\{S(T)<K, M^{S}(T)<L\right\}}\right]=N\left(-a_{1}\right)-\frac{S(t)}{L} N\left(-a_{2}\right)
$$


4. If $S<L$ and $t<\tau_{L}$ then

$$
\widetilde{E}_{t}\left[\mathbb{1}_{\left\{\tau_{H}>T\right\}}\right]=\widetilde{E}_{t}\left[\mathbb{1}_{\left\{M^{S}(T)<L\right\}}\right]=N\left(-b_{1}\right)-\frac{S(t)}{L} N\left(-b_{2}\right)
$$

$\underline{\text { Proof }}$

1. Consider a down-and-out binary call (DOBC) with strike price $K$, barrier $L$ and maturity $T$. The payoff of this option is $\mathbb{1}_{\left\{S(T)>K, m^{S}(T)>L\right\}}$. The price of this contingent claim $\operatorname{DOBC}(t)$ is nonzero if $t<\tau_{L}$ and zero if $t>\tau_{L}$.

Consider now a portfolio that consists in a long position of one binary call with strike $K$ and maturity $T$ and a short position of $\frac{1}{L}$ gap puts with strike price $\frac{L^{2}}{K}$ and maturity $T$. It can be seen from Lemma 2.1 that if $t=\tau_{L}$ (i.e. $S(t)=L$ ) the price of this portfolio is zero. So if the barrier is hit previous to the maturity $T$ the value of this portfolio is equal to that of the option. If the barrier is not hit the portfolio and the option will have the same payoff at maturity since the gap put is out of the money $\left(S(T)>L, K>L \Rightarrow S(T)>\frac{L^{2}}{K}\right.$ ).

Since the fractional Black-Scholes does not have arbitrage and the down-andout binary call and the portfolio have the same payoff, their value will be the same for $t<\tau_{L} \wedge T$. So

$$
D O B C(t)=B C(t)-\frac{1}{L} G P(t)
$$

2. Consider a contingent claim that pays one unit if the stock price does not hit the barrier $L$ before $T$ (down-and-out bond). The payoff of this contingent claim is ${ }_{\left\{m^{S}(T)>L\right\}}$. As in the previous case we look for a portfolio that has the same value as the contingent claim at $\tau_{L} \wedge T$, and as a consequence of the no-arbitrage property of the fractional Black-Scholes they will have the same value for every $t<\tau_{L} \wedge T$.

In this case we can chose a portfolio consisting in a long position of one binary call with strike $L$ and maturity $T$ and a short position of $\frac{1}{L}$ gap puts with strike price $L$ and maturity $T$.

3. In this case we consider an up-and-out binary put and a portfolio that consists in a long position of one binary put with strike $K$ and maturity $T$ and a short position of $\frac{1}{L}$ gap calls with strike price $\frac{L^{2}}{K}$ and maturity $T$. 
4. In this case we consider an up-and-out bond and a portfolio that consists in a long position of one binary put with strike $L$ and maturity $T$ and a short position of $\frac{1}{L}$ gap calls with strike price $L$ and maturity $T$.

\section{Theorem 2.2}

Consider that $r=0$.

1. If $K>L, S>L$ and $t<\tau_{L}$ then

$$
\widetilde{E}_{t}\left[S(T) 1_{\left\{S(T)>K, m^{S}(T)>L\right\}}\right]=S(t) N\left(c_{1}\right)-L N\left(-c_{2}\right)
$$

where $c_{1}=\frac{\ln \left(\frac{S(t)}{K}\right)+\frac{\sigma^{2}}{2}\left(T^{2 H}-t^{2 H}\right)}{\sigma \sqrt{T^{2 H}-t^{2 H}}}$ and $c_{2}=\frac{\ln \left(\frac{K S(t)}{L^{2}}\right)-\frac{\sigma^{2}}{2}\left(T^{2 H}-t^{2 H}\right)}{\sigma \sqrt{T^{2 H}-t^{2 H}}}$

2. If $K<L, S<L$ and $t<\tau_{L}$ then

$$
\widetilde{E}_{t}\left[S(T) \mathbb{1}_{\left\{S(T)<K, M^{S}(T)<L\right\}}\right]=S(t) N\left(-c_{1}\right)-L N\left(c_{2}\right)
$$

$\underline{\text { Proof }}$

1. Consider a down-and-out call with strike price $K$, barrier $L$ and maturity $T$. The payoff of this option is $(\mathrm{S}(\mathrm{T})-K) 1_{\left\{S(T)>K, m^{S}(T)>L\right\}}$. The value of this option at $t<\tau_{L} \wedge T$ is given by:

$$
\widetilde{E}_{t}\left[S(T) 1_{\left\{S(T)<K, M^{S}(T)<L\right\}}\right]-K \widetilde{E}_{t}\left[\mathbb{1}_{\left\{S(T)<K, M^{S}(T)<L\right\}}\right]
$$

One can see that this contingent claim has the same payoff as a portfolio that consists in a long position of one call with strike $K$ and maturity $T$ and a short position of $\frac{K}{L}$ puts with strike price $\frac{L^{2}}{K}$ and maturity $T$. Using Theorem 4.2 in Necula (2002), the value of this portfolio at $t<\tau_{L} \wedge T$ is:

$$
S(t) N\left(c_{1}\right)-L N\left(-c_{2}\right)-K\left(N\left(a_{1}\right)-\frac{S(t)}{L} N\left(a_{2}\right)\right)
$$


2. In this case we consider an up-and-out put and a portfolio that consists in a long position of one put with strike $K$ and maturity $T$ and a short position of $\frac{K}{L}$ calls with strike price $\frac{L^{2}}{K}$ and maturity $T$.

We know that there is a probability measures $P^{*}$ such that $B_{H}^{*}(t)=-\frac{\sigma}{2} t^{2 H}+B_{H}(t)$ is a fBm under $P^{*}$.

The reflection principle of the Brownian motion gives the common distribution of the Brownian motion and its minimum (or maximum).

The next corollary gives a similar result for the fractional Brownian motion.

\section{Corollary 2.1}

1. If $y \leq 0, x>y$ and $t<\tau_{y}$ then

$$
\begin{aligned}
\widetilde{E}_{t}\left[{ }_{\left\{B_{H}^{*}(T)>x, m^{B_{H}^{*}}(T)>y\right\}}\right]=N\left(\frac{B_{H}^{*}(t)-x+\frac{\sigma}{2}\left(T^{2 H}-t^{2 H}\right)}{\sqrt{T^{2 H}-t^{2 H}}}\right)- \\
-\exp \left\{\sigma\left(B_{H}^{*}(t)-y\right)\right\} N\left(\frac{2 y-x-B_{H}^{*}(t)+\frac{\sigma}{2}\left(T^{2 H}-t^{2 H}\right)}{\sqrt{T^{2 H}-t^{2 H}}}\right)
\end{aligned}
$$

2. If $y \geq 0, x<y$ and $t<\tau_{y}$ then

$$
\begin{array}{r}
\widetilde{E}_{t}\left[{ }_{\left\{B_{H}^{*}(T)<x, M^{B_{H}^{*}}(T)<y\right\}}\right]=N\left(\frac{x-B_{H}^{*}(t)-\frac{\sigma}{2}\left(T^{2 H}-t^{2 H}\right)}{\sqrt{T^{2 H}-t^{2 H}}}\right)- \\
-\exp \left\{\sigma\left(B_{H}^{*}(t)-y\right)\right\} N\left(\frac{x-2 y+B_{H}^{*}(t)-\frac{\sigma}{2}\left(T^{2 H}-t^{2 H}\right)}{\sqrt{T^{2 H}-t^{2 H}}}\right)
\end{array}
$$

The shortcoming of the result is that the quasi-conditional expectation it is under $P$, not under the probability measure $P^{*}\left(B_{H}^{*}(t)\right.$ is a $\mathrm{fBm}$ under $P^{*}$, not under $P)$. 


\section{$\underline{\text { Remarks }}$}

For finance an important result would be a formula for the price of barrier options in the case in which the interest rate is not zero. But the extension to the case of non-zero drift in 2.1 seems very difficult.

\section{References}

Alos, E., O. Mazet and D. Nualart, (2001), Stochastic calculus with respect to Gaussian processes, Annals of Probability, 29, 766-801

Alos, E. and D. Nualart, (2002), Stochastic calculus with respect to the fractional Brownian motion, Preprint, University of Barcelona

Bender, C. (2002) The Fractional Ito Integral, Change of Measure and Absence of Arbitrage, Preprint

Bender, C. (2003) Explicit Solutions of a Class of Linear Fractional BSDEs and Applications to Finance, Preprint

Decreusefond, L and A.S. Ustunel, (1999), Stochastic analysis of the fractional Brownian motion, Potential Analysis, 10, 177-214

Duncan, T. E., Y. Hu and B. Pasik-Duncan, (2000), Stochastic calculus for fractional Brownian motion, I. Theory, SIAM J. Control Optim., 38, 582-612.

$\mathrm{Hu}, \mathrm{Y}$. and B. Oksendal, (2000), Fractional white noise calculus and application to Finance, Preprint, University of Oslo

Hu, Y., B. Oksendal, and A. Sulem, (2000), Optimal consumption and portfolio in a Black-Scholes market driven by fractional Brownian motion, Preprint 23/2000, University of Oslo

Lin, S.J. (1995), Stochastic analysis of fractional Brownian motion, fractional noises and applications, SIAM Review, 10, 422-437.

Necula, C. (2002), Option Pricing in a Fractional Brownian Motion Environment, working paper, DOFIN, Academy of Economic Studies, Bucharest

Necula, C. (2003), Stochastic Calculus of the Fractional Brownian Motion and Applications in Mathematical Finance, MSc Dissertation Paper, Faculty of Mathematics, University of Bucharest

Oksendal, B (2003), Fractional Brownian motion in finance, Preprint 28/2003, University of Oslo 
Perez-Abreau, V. and C. Tudor, (2002), A Transfer Principle for Multiple Stochastic Fractional Integrals, Preprint, CIMAT

Rogers, L.C.G. (1997), Arbitrage with fractional Brownian motion, Mathematical Finance, 7, 95-105

Tudor, C. (2002), On the Wiener integral with respect to the fractional Brownian motion, Bol. Mex. Mat. Soc, 8, 97-106 